\title{
Una revisión bibliográfica de los movimientos estudiantiles en España, México y Colombia (1968-1971)i
}

\author{
A literature review of the student movements in Spain, Mexico \\ and Colombia (1968-1971)
}

Tânia Gorayeb Sucupira

Orcid: http://orcid.org/0000-0001-8087-7651

Universidade Federal do Ceará, Fortaleza, Brasil, thaniasucupira@yahoo.com.br

\section{Francisco Javier García-Delgado \\ Orcid: http://orcid.org/0000-0001-8863-4179 \\ Universidade de Huelva - Departamento de \\ História, Geografia e Antropologia - Faculdade de \\ Ciências de Negócios e Turísmo, Huelva, Espanha, fcogarci@uhu.es}

\section{José Gerardo Vasconcelos}

Orcid: http://orcid.org/0000-0003-0559-2642

Universidade Federal do Ceará, Fortaleza, Brasil, gerardovasconcelos1964@gmail.com

\section{DOI: 10.21680/2596-0113.2021v4n0ID26868}

Citation: Sucupira, T. G.; García-Delgado, F. J. \& Vasconcelos, J. G. (2021). Una revisión bibliográfica de los movimientos estudiantiles en España, México y Colombia (1968-1971). History of Education in Latin America - HistELA, 4, e26868.

Competing interests: The authors have declared that no competing interests exist.

Editor: Olivia Morais de Medeiros Neta

Received: 08/10/2021

Approved: 29/10/2021

OPEN ACCES

\section{Resumen}

Desde finales de los años 1960 se da una oleada de movimientos estudiantiles por todo el mundo. En los casos de España, México y Colombia estos movimientos se concentran entre 1968 y 1971. El objetivo es comprender las circunstancias que rodean situaciones críticas, entre factores, elementos y desarrollos, basada metodológicamente en una revisión bibliográfica para la construcción de la investigación. Los resultados obtenidos han sido: a) en España, la acción política y coordinada de los estudiantes universitarios es uno de los elementos que anticipa el escenario democrático futuro, pero no supone un cambio importante a corto plazo en la universidad española; b) en México, la represión (masacre de Tlatelolco) pone fin a las protestas contra la política autoritaria de Díaz Ordaz, sin suponer un cambio real de la situación previa; c) en Colombia, el movimiento estudiantil dio como resultado el Programa Mínimo para Estudiantes Colombianos, con una breve experiencia, sin precedentes, del cogobierno en la toma de decisiones en los Consejos Superiores. En conclusión, compartiendo parte de los factores causantes, en contextos diferentes, los movimientos estudiantiles consiguieron solo en parte sus objetivos, siendo fundamental en el análisis atender a sus singularidades (contextuales y conceptuales).

Palabras clave: Historia de la educación. Contracultura. Democracia. Movimientos estudiantiles.

\section{Abstract}

Since the late 1960s there has been a wave of student movements around the world. In the cases of Spain, Mexico, and Colombia these movements are concentrated between 1968 and 1971. The objective is to understand the circumstances surrounding critical situations between factors, elements, and development methodologically based on a bibliographic review for the construction of the research. The results obtained have been a) in Spain the political and coordinated action of university students is one of the elements that anticipates the democratic future scenario, But it does not represent an important change in the short term in the Spanish university; b) in Mexico the repression (the Tlatelolco massacre) puts an end to the protests against the authoritarian policy of Díaz Ordaz, without assuming a real change from the previous situation; c) in Colombia the student movement resulted in the Minimum Program for Colombian students with a brief unprecedented experience of the co-government in decision-making in the Superior Councils. In conclusion, sharing part of the causative factors in different contexts, student movements only partially achieved their goals, being fundamental in the analysis to attend to their singularities (contextual and conceptual).

Keywords: Education History. Counterculture. Democracy. Student movements 


\section{Introducción}

El final de la década de los 60 y principios de los 70 marcan los movimientos estudiantiles en todo el mundo (Carrillo-Linares, 2015), aunque los más conocidos sean el Mayo Francés (1968) y la Primavera de Praga (1968) (Fuentes, 2008), con implicaciones estudiantiles pero un marcado carácter de reacción y contracultura, hubo otros muchos movimientos, que solo entre 1968 y 1969 afectaron a más de 50 países (Carrillo-Linares, 2015), con todo tipo de regímenes políticos: democracias (con gobiernos de derechas -muchas de ellas con derivas autocráticas - e izquierdas) y totalitarismos (de derecha e izquierda o monarquías absolutas) (Mapa 1).

Mapa 1. Países en los que se registran movimientos estudiantiles (1968-1969)



Elaboración propia a partir de Carrillo-Linares, 2015.

A la hora de abordar los estudios sobre los movimientos estudiantiles de los años sesenta y setenta del siglo XX, los diferentes autores toman en consideración diferentes factores y elementos con los que la contestación social (en forma de movimiento estudiantil) se relaciona:

1) El contexto internacional: la Guerra Fría y el enfrentamiento entre los bloques capitalista y socialista, que se manifiesta dentro de cada uno de ellos en los "anti" (anticomunismo, anti-capitalismo...) y sus resultados (recorte de las libertades democráticas, golpes de estado...) (Suárez-Inclán \& Gómez-Acebo, 2021).

2) El contexto nacional: cada país tiene su propio contexto específico político (régimen y oposición al mismo), socio-cultural (cultura dominante y contracultura) y económico (auge o crisis) (Amézquita-Zárate, 2010; Sierra-Garzón, 2015).

3) El marco institucional: las normas (vinculadas al contexto político) que rigen en la Universidad/Enseñanza y el tipo de institución (pública, privada, central, autónoma...), el acceso a la formación universitaria (cuantitativo y cualitativo) (Marini, 1970).

4) Los factores causantes: coyunturas que desencadenan el movimiento (cambio legal, destitución de profesorado, tensión entre estudiantes y autoridades universitarias ...). (Cejudo-Ramos, 2020; Díaz-Jaramillo, 2021).

5) La organización del movimiento: de forma más o menos espontánea u organizada, con fuertes vinculaciones políticas (o sociales) o como resultado de una conciencia de clase (estudiantil), en acciones aisladas o generales (Flores-Padilla, 2021). 
6) Los métodos de lucha estudiantiles: ocupaciones de centros, manifestaciones, difusión de escritos, etc (Suárez-Inclán \& Gómez-Acebo, 2021).

7) Los partidarios y detractores: sociales y políticos, pero también la manifestación en la prensa periódica (como medio de masa en la época) (Serna, 2014; HernándezRamos, 2017).

8) El desarrollo: las fases del movimiento desde que aparece hasta que desaparece. (Suárez-Inclán; Gómez-Acebo, 2021).

9) Los resultados: la forma de concluir el movimiento (revolución, represión, pacto, extinción silenciosa...) (Dávila, 2021).

10) Las consecuencias: inestabilidad social, procesos de represión y privación de libertad (deriva autoritaria y golpes de estado), apertura democrática, creación de conciencia política, etc) (Castillo-Troncoso, 2008; Díaz-Jaramillo, 2021; Suárez-Inclán \& Gómez-Acebo (2021).

En líneas con trabajos anteriores (Sucupira et al., 2019; Sucupira et al., 2020) este estudio pretende abordar de manera comparada los movimientos estudiantiles entre 1968 y 1971 en tres países iberoamericanos: España, México y Colombia, ensamblado como marco teórico para su posterior comparación con Brasil en la Tesis de Doctorado (Sucupira, 2021).

\section{Metodología}

La presente investigación es cualitativa, exploratoria y descriptiva y se basa en la revisión exhaustiva de la literatura científica publicada, siguiendo el análisis de tres elementos de los movimientos estudiantiles (Marini, 1970; Sucupira \& García-Delgado, 2020): a) los contextos (político, socio-cultural y económico) en los que se dan; b) las causas que los desencadenan; c) los desarrollos que los singularizan.

Una vez determinados los elementos a estudiar, la búsqueda a través de Bases de Datos de la Biblioteca de la Universidad de Huelva (<https://guiasbuh.uhu.es/az.php>) y Google Scholar@ (<https://scholar.google.com/>) se centró en: «movimientos estudiantiles» y «agitación estudiantil» en España, México y Colombia, acontecidos en la ventana temporal 1968-1971. Con el fin de conseguir una visión historiográfica completa, se restringió la búsqueda a publicaciones del período 2000-2020. Las búsquedas se realizaron en español ${ }^{3}$.

En correspondencia con las líneas de investigación del Doctorado en Educación, se restringió el estudio a trabajos que utilizasen la prensa periódica como una fuente importante de investigación historiográfica (HernándezRamos, 2017), pues se trata de "Pensar al movimiento estudiantil más allá de la primera página de los periódicos, aproximándolo a cuestiones más profundas y complejas [...]' (Acevedo-Tarazona y Samacá-Alonso, 2011: 112). De esta forma, se seleccionaron los siguientes trabajos:

- España: Carrillo-Linares, 2006; Carrillo-Linares, 2008; BadenesSalazar, 2018.

- $\quad$ México: Allier-Montana, 2009; Delgado, 2013.

- Colombia: Acevedo-Tarazona, 2015; Sosa-Londoño, 2018.

Estas obras, analizadas sistemáticamente, se complementan con otros trabajos científicos (publicaciones en revistas, tesis, ensayos y monografías), a 
la vez que se toman referencias directas de la prensa periódica de la época a la que se ha podido acceder a través de las hemerotecas digitales, que reflexionaban sobre contextos críticos y situaciones relevantes para el fenómeno social y estudiantil. A partir de los trabajos se realiza un análisis textual y discursivo, aplicando un método crítico-dialéctico (Alyrio, 2009) para comprender las interrelaciones de factores y elementos en los respectivos movimientos estudiantiles y "en su carácter comparado" (Suárez-Inclán \& Gómez-Acebo, 2021: 7).

\section{Desarrollo}

\section{El movimiento estudiantil en España (1968)}

España vivía en una dictadura militar conservadora desde 1939 (CarrilloLinares \& Rodríguez-Tejada, 2019), cuando en 1968 los estudiantes universitarios españoles organizaron una acción política para reclamar democracia y una mayor participación en las instancias deliberativas universitarias (Carrillo-Linares, 2018). Es en un contexto de cierta "permisividad", conocida como "dictablanda" del régimen franquista, pero que sigue careciendo de libertad (Feixa \& Porzio, 2004), en el que las clandestinas ideas democráticas (desde la derecha hasta la izquierda) comienzan a imponerse en la intelectualidad del país.

En España existían en 1968 un total de 11 universidades públicas (solo la Universidad de Murcia se crea en 1915, el resto anteriores a 1600) y 4 privadas concordadas (preexistentes, son todas católicas y se atienen al Concordato con la Santa Sede de 1953), si bien existían campus, escuelas y centros en diferentes ciudades, además de centros adscritos y privados. El número de estudiantes universitarios conoció un importante incremento a lo largo de los años sesenta, pasando de 170.602 en el curso 1960-1961 a 346.027 en 19691970 (Rahona-López, 2008: 40), es decir, el porcentaje de universitarios sobre la población total pasó del $0,56 \%$ al $1,04 \%$ en solo 10 años.

Los universitarios españoles, al igual que los de otras partes del mundo, eran "[...] jóvenes de indumentaria hippie, inconformistas que entraban en contacto con el marxismo y las [...] teorías incendiarias [...]' (Carrillo-Linares, 2008: 86) y ya no encajaban en las estructura del Sindicato Español Universitario (SEU, parte del Sindicato Vertical organizado por el franquismo) (Rodríguez-Tejada, 2011). El activismo estudiantil se va a dar por el anhelo de democracia (participación), el descontento con el currículum (desactualizado basado en grandes bloques de conocimiento con especializaciones- y rígido) y los problemas de gestión institucional (centralizada y politizada desde el Movimiento -nombramiento de rectores desde las instancias gubernativas, con afinidad probada al régimen-), sin participación del estamento estudiantil (más allá del SEU). La politización estudiantil es combatida por esas instancias, utilizando la "inteligencia" (infiltrados) para castigar la rebelión y perseguir a los militantes (Badenes-Salazar, 2018).

La ruptura con el franquismo se produciría en las grandes universidades, en Madrid y Barcelona (Ysàs, 2007), respondiendo a un malestar estudiantil generalizado desde 1964-1965 (Carrillo-Linares, 2008), que derivó en la 
expulsión de profesores de la Universidad española a perpetuidad tras manifestar su apoyo a los estudiantes, pero también en el fin del SEU (1965), siendo esta "la primera y única vez durante todo el franquismo que un movimiento de oposición abierta [el estudiantil] conseguía acabar con una institución del régimen" (Carrillo-Linares, 2015: 57). Que los estudiantes universitarios se organizaran de forma autónoma sería solo cuestión de tiempo.

En 1966, el Sindicato Democrático de Estudiantes Universitarios de Barcelona (SDEUB) institucionalizó el proceso de constitución: los delegados de las facultades gestionaron la reforma en las Reuniones Coordinadas Preparatorias (RCP) y el Congreso Nacional de Estudiantes certificó la constitución del nuevo Sindicato Democrático de Estudiantes Universitarios (SDEU) (Carrillo-Linares, 2008). La IV RCP tuvo lugar en Madrid entre el 10 y el 12 de octubre de 1967, con delegados de las Universidades de Barcelona, Sevilla y Santiago de Compostela. A principios de noviembre, los disturbios coincidieron "[...] con una llamada a la huelga nacional efectuada por las

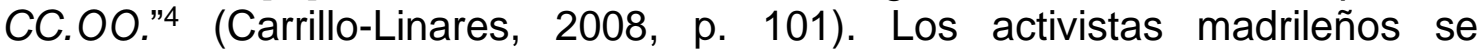
enfrentaron a la Policía Armada y sus perros adiestrados, pero la V RCP extraordinaria en Madrid llegó a la conclusión de acelerar la constitución de los sindicatos universitarios y llevar a cabo la representación nacional, es decir, el Sindicato Democrático de Estudiantes de España (SDEE) (Carrillo-Linares, 2008), con el fin de defender los intereses estudiantiles.

Los estudiantes denunciaban la falta de medios económicos de las Universidades, además, insistían en la desconexión del currículum universitario de las demandas de la sociedad. Los estudiantes se limitaban a "contemplar" la sociedad, es decir, "estudiar" (Argullol-Murgadas, 1977, p. 86), despojados de toda actitud crítica. Por otra parte, inciden en la inadecuación docente por "[...] el carácter vitalicio de las cátedras. Especialmente cuando esos profesores no cumplían bien con su deber. Llegaban tarde a clases o [...] no iban [...] incluían materia que no habían explicado [...]' (Badenes-Salazar, 2018, p. 191).

El pleno de diciembre de 1967 deliberó sobre el Sindicato de Estudiantes de la Universidad de Sevilla, y Camilo Tejera, de la Facultad de Derecho, entregó el documento del sindicato (en la línea del sindicato madrileño) al Rector Dr. José Antonio Calderón Quijano ${ }^{5}$, quien cuestionó, desde enero de 1968, la validez de la reclamación. La prensa escrita, en mayo, ratificó los argumentos del Rector: "[...] uno de los vestíbulos de la Facultad había prendidos de las paredes varios carteles injuriosos, cuyos textos reprobaban la mayoría de los estudiantes" (ABC DE MADRID 6 , 8 de mayo de 1968).

El 21 de enero de 1968, se inicia la huelga estudiantil universitaria en Madrid, durando cinco días. El día 3 de febrero, en la Facultad de Filosofía y Letras de la Universidad de Sevilla se distribuyen boletines y carteles convocando para una asamblea de estudiantes, cuya realización fue prohibida. El 21 de febrero, el profesor Dr. Jesús Arellano Catalán, catedrático de Fundamentos de Filosofía, propuso al Decano de esta facultad la representación democrática para resolver el impasse (Carrillo-Linares, 2008). El Gobierno respondió con más reglas para disciplinar la conducta y el uso fuerza represiva específica contra los estudiantes, la Policía de Orden Universitario, que había sido creada en enero de 1968 (Carrillo-Linares \& Cardina, 2012). 
Así, se inicia en la Universidad de Sevilla "el mayo que fue en marzo" (Carrillo-Linares, 2008, p. 133), fatídico para el movimiento estudiantil sevillano. La VI RCP se llevó a cabo con manifestaciones para la formación del Sindicato en la Universidad de Sevilla, para componer el cuerpo de representación nacional de estudiantes (SDEE). El punto álgido de la subversión se produce a partir del 26 de marzo, cuando alumnos de diversas facultades y escuelas se concentraron en la Facultad de Derecho de la Universidad de Sevilla y se dirigieron al Rectorado con carteles exigiendo libertad de expresión, reunión y asociación (Carrillo-Linares, 2008). En las calles, los estudiantes impedían la circulación al grito de: "[...] 'abajo la dictadura', 'libertad', 'democracia' o 'Universidad para los obreros" (Carrillo-Linares, 2008, p. 143). La fuerza policial se acercó con toques de corneta, reprimiendo la manifestación, e incriminó a un grupo de ocho estudiantes acusados de alterar el orden público, pero los activistas articularon estrategias (Carrillo-Linares, 2008). En los planes de los movimientos, un grupo atraía a la fuerza policial, mientras que otros se movían por las calles, ampliando el radio y la fuerza de la acción estudiantil, serpenteando por las calles gritando consignas, manifestándose y dispersándose para confundir a la policía. El grupo que se había reunido en la Facultad de Derecho escapó de la policía, porque los estudiantes captaron la comunicación y alertaron a sus compañeros (Carrillo-Linares, 2008).

Ante la situación, se reunió el Consejo de Ministros presidido por Franco el 29 de marzo, para evaluar el avance de la resistencia estudiantil (CarrilloLinares, 2008, p. 147). El día 30 de marzo, una nota del Gobernador Civil de Sevilla, José Utrera Molina, informó la sanción impuesta a Camilo Tejera y a otros 35 jóvenes, quienes fueron condenados a pagar una multa por la alteración de orden público los días 27, 28 y 29 (ABC DE MADRID, 30 de marzo de 1968). Por su parte, la Junta de Gobierno de la Universidad aplicó sanciones a 23 estudiantes, con penas que iban desde la suspensión de la matrícula por dos cursos consecutivos hasta la expulsión definitiva (a perpetuo) y la prohibición de entrada en los centros (ABC DE MADRID, 31 de marzo de 1968). La Universidad de Santiago de Compostela (USC) lanzó un paro en solidaridad con los compañeros castigados, y la oficina de prensa de la Universidad de Barcelona pidió a sus estudiantes la normalidad, amenazando actos de indisciplina con la "[...] correcta aplicación que el Reglamento de Disciplina Universitaria" (ABC DE MADRID, 2 de abril de 1968). El 3 de mayo de 1968, $A B C$ de Madrid y La Vanguardia de Barcelona informaron de la suspensión de sanciones a los 13 universitarios en la USC, pero la prensa insistió en la crisis, con los estudiantes de la Facultad de Farmacia aún con "paro académico" (ABC DE MADRID, 3 de mayo de 1968).

En junio de 1968, el Gobierno crea las Universidades Autónomas de Madrid y Barcelona, segregadas de sus respectivas Universidades Centrales, como sucede en 1971 con las Universidades Politécnicas de Barcelona, Madrid y Valencia. En 1972 son creadas 3 universidades (Cantabria, Córdoba y Málaga) y en 1973 la última del franquismo (Extremadura). 


\section{El movimiento estudiantil en México (Universidad Nacional Autónoma de México e Instituto Politécnico Nacional) (1968)}

En México se suceden entre 1958 y 1970 dos presidentes del Partido Revolucionario Institucional (PRI) ${ }^{7}$ : Adolfo López Mateos (1958-1964) y Gustavo Díaz Ordaz (1964-1970). El primero nombró un gabinete de tecnócratas y desarrollista en los económico (Hansen, 1971), pero puso al cargo de la Secretaría de Gobernación al que sería su sucesor en la Presidencia de la República, quien reprimió duramente los movimientos obreros (empleados de ferrocarriles) y de maestros (Movimiento Revolucionario del Magisterio), siendo sus líderes encarcelados, o asesinados (Meyer, 2013). El gobierno de Díaz Ordaz seguiría con la represión en un contexto desarrollista en lo económico, en el que las desigualdades sociales se intensificaron (Gollás, 2003). La violencia institucional contra los grupos disidentes estaba servida (Meyer, 2013).

En 1959 triunfa la Revolución Cubana, su éxito renueva el ideal comunista en toda Latinoamérica, y el espíritu revolucionario (Massholder, 2018). El ideal comunista prende entre la juventud mexicana, que ve cómo su país se convierte en expresión de las distorsiones del capitalismo: concentración de la riqueza y exclusión social (Delgado, 2013).

En el caso mexicano, la estructura universitaria es compleja, combinándose Universidades federales, con Universidades estatales, Institutos Tecnológicos y Escuelas Normales y otras instituciones universitarias. Con una estructura heredada de la época colonial, el mapa universitario se reestructura a partir de 1910 con la creación de la Universidad Autónoma de México (UNAM) (heredera de la Real y Pontificia Universidad de México, creada en 1551) (Marsiske, 2006). Entre 1950 y 1970, el número de estudiantes universitarios pasó en México de 30.000 a 200.000 (Taborga-Torrico, 2003), aunque debido al incremento de la población la cobertura siguió siendo deficiente (Donoso-Romo, 2016) supuso pasar del $0,11 \%$ de la población al $0,39 \%$.

En un sistema universitario fuerte (enriquecido por la intelectualidad del exilio español) no tarda en surgir una organización estudiantil: el Centro Nacional de Estudiantes Demócratas (CNED) (1963), apoyado por el Partido Comunista Mexicano (PCM). Pese a reunir a estudiantes de todas las ideologías democráticas, fortaleciendo las federaciones regionales y ampliando el alcance de la acción del partido, así "Tanto la CNED como la JCM [Juventud Comunista Mexicana] tomaron fuerza durante los años 1962-1964" (Delgado, 2013: 54). El primer enfrentamiento del CNED con el Gobierno se produce por la oposición al nombramiento de Díaz Ordaz, al tiempo que se crean una serie de federaciones estudiantiles regionales del CNED "que denunciaban el endurecimiento del sistema político y socioeconómico mexicano, y demandaban mayor libertad política y académica, protestaban contra las federaciones estudiantiles oficialistas del PRI, y vinculaban las acciones de la juventud a las luchas de los obreros y campesinos, siempre con un discurso antimperialista" (Delgado, 2013, p. 49). 
La crisis de 1968 comenzó con los enfrentamientos el 22 de julio entre los estudiantes de las Vocacionales 2 y 5 del Instituto Politécnico Nacional (IPN) y los de la preparatoria Isaac Ochoterena: escuelas privadas y preparatorias de educación superior, pero vinculadas a la UNAM. Al día siguiente, la policía intervino, resultando varios estudiantes heridos (Allier-Montana, 2009). Díaz Ordaz, con el acuerdo de agentes de la Dirección Federal de Seguridad (DFS), denunció que los conflictos eran parte de "[...] una 'conjura', proveniente del Partido Comunista Mexicano (PCM) y otras organizaciones de izquierda" (Allier-Montana, 2009, p. 293) para boicotear los Juegos Olímpicos que se celebrarían en México en octubre.

La prensa fortaleció la opinión pública en contra los jóvenes comunistas, apoyada por sectores de la Iglesia Católica, la elite económica y organizaciones de derecha. Sin embargo, no hubo evidencia concreta de la acusación, y la declaración del periodista y activista del PCM al Canal el 6 de julio de 1968 en el programa «La Conexión Americana» aclaró los hechos:

Se ha dicho que el PC era muy activo, sí es cierto, pero quizá seríamos mil quinientos, dos mil militantes jen todo el país!, entonces hablar de que nos podíamos levantar en armas, que podíamos hacer una revolución e incluso una revuelta era una locura verdadera. (Delgado, 2013, p. 70).

El argumento tenía sentido. En un país con casi 48,5 millones de habitantes, sería muy difícil que 2,000 militantes comunistas llevaran a cabo una revolución, pero las autoridades peinaron al Comité Central del PCM en busca de pruebas, mientras que los líderes del partido aseguraron que los conflictos del 22 y 26 de julio fueron "[...] producto de toda una provocación gubernamental en contra de los militantes del PCM"' (Delgado, 2013, p. 73).

Las cabeceras de prensa periódica (El Universal, Novedades, El Heraldo de México, El Sol de México, Excélsior y El Día), con tendencias que oscilaban de la derecha a la izquierda, pero que siempre "mantuvieron una relación estable con el Estado priísta" (Serna, 2014, p. 125) agitaban a la ciudadanía en contra del peligro comunista, "embebidos de la paranoia anticomunista de la época" (Serna, 2014, p. 125) tras la Revolución Cubana. Así, en las portadas aparece un ataque frontal al movimiento estudiantil. Inicialmente, el más crítico con el gobierno fue el priísta El Día, que sufrió una división en la redacción, para volver al apoyo gubernamental (Serna, 2014, p. 129). El 28 de julio, El Heraldo de México ${ }^{8}$ publica el titular "Energía contra los alborotadores" y sigue "Represión inmediata de Disturbios y Escándalos. Que los Padres de Familia cuiden de sus Hijos estudiantes, pide el General Cueto [Jefe de la Policía del Distrito Federal]". Como señala Alberto del Castillo-Troncoso, en esta publicación contrasta el apoyo al Gobierno con la moderna cobertura dada por el medio y su multiplicidad de miradas (del Castillo-Troncoso, 2008, p. 72).

Los siguientes hechos ocurrieron de manera vertiginosa. Los estudiantes ocuparon los Preparatorios 1, 2 y 3 de la UNAM en la madrugada del 27 de julio y resistieron hasta el día 29, cuando granaderos y policías militares armados tomaron los edificios y detuvieron a los insurgentes. El 30 de julio, el PCM publicó una nota repudiando el autoritarismo del gobierno en la revista marxista Historia y Sociedad, y Javier Barros Sierra, Rector de la UNAM, respondió con una bandera a media asta en la Ciudad Universitaria "[...] en señal de luto por la violación de la autonomía en los días anteriores" (Delgado, 2013, p. 78). 
Se creará un Consejo Nacional de Huelga $(\mathrm{CNH})$, compuesto por delegados de escuelas, miembros de la JCM, el CNED, la Federación de Campesinos Socialistas de México y el PCM (Delgado, 2013). Reclamaron la libertad de los presos políticos, la derogación de las leyes de disolución social y acercamiento a la población civil, la extinción del Cuerpo de Granaderos, la destitución de los jefes policiales, la indemnización a los familiares de los muertos y heridos en los conflictos y la rendición de cuentas de los responsables de la violencia contra los estudiantes (Delgado, 2013).

EI Rector Barros Sierra de la UNAM estuvo presente en el encuentro de principios de septiembre de 1968, en Tlatelolco, cuando declaró el fin de los enfrentamientos políticos y suplicó a la comunidad que pusiera fin a las disputas, alegando que se atendieron los reclamos, según él, "[.... En lo esencial, de la Ciudadano Presidente de la República, en su último informe" (Delgado, 2013, p. 102). El CNH respondió al Rector el 13 de septiembre con la "Manifestación del Silencio": "[...] con 100 mil ciudadanos amordazados" (Fuentes, 2008, p. 145).

El 19 de septiembre, tropas del ejército ocuparon la Ciudad Universitaria de la UNAM y detuvieron a más de 100 estudiantes y el 23 de septiembre de 1968 el Rector entregó su renuncia, que no fue aceptada (Delgado, 2013). El 30 de septiembre, estudiantes y policías se enfrentaron en el Casco de Santo Tomás, donde se sitúa la Unidad Profesional Lázaro Cárdenas (IPN), dejando muertos y el edificio ocupado por el ejército (Delgado, 2013). Al día siguiente, la asamblea de la Rectoría de la Ciudad Universitaria denunció las ejecuciones sumarias, citando las muertes del día anterior y la huelga de hambre de los presos políticos (Delgado, 2013).

El fatídico 2 de octubre de 1968 comenzó con una reunión entre representantes gubernamentales y miembros del $\mathrm{CNH}$. Por la tarde, el $\mathrm{CNH}$ elige cuatro oradores para dirigirse a los estudiantes, tres de los cuales formaban parte de la JCM (Delgado, 2013). La masacre tuvo lugar en la Plaza de las Tres Culturas, sección de Tlatelolco (Allier-Montana, 2009; Delgado, 2013; Fuentes, 2008). Según Fuentes (2005, p. 145), miles de mexicanos masacrados (cientos muertos y miles heridos) y la revuelta de las víctimas que sobrevivieron a la masacre de Tlatelolco, ante el desprecio de las autoridades:

Nadie tiene derecho a reconocer un cadáver. Nadie tiene derecho a transportar a una persona muerta. No habrá quinientas procesiones fúnebres en esta ciudad mañana. Lanza los cadáveres a la fosa común. Que nadie los reconozca. Desaparece con ellos. (Fuentes, 2008, pp. 147-148).

El 12 de octubre de 1968, en la Ciudad de México, Gustavo Díaz Ordaz inauguró alegremente los XIX Juegos Olímpicos con una bandada de palomas de la paz (Fuentes, 2008; Jiménez-Guzmán, 2011). En 1971, el nuevo presidente Luis Echeverría Álvarez, secretario de Gobernación de Díaz Ordaz, en 1968, anunció una apertura democrática, otorgando libertad a los activistas sociales detenidos en 1968 (Allier-Montana, 2009). 

1971)

El escenario político en Colombia es diferente. Los movimientos estudiantiles se dan en un régimen democrático "a la colombiana" dentro del período del Frente Nacional (alianza turnista entre liberales y conservadores entre 1958 y 1974), se suceden el gobierno liberal de Carlos Alberto Lleras Restrepo (1966-1970) y el del conservador Misael Pastrana Borrero (19701974). Estos gobiernos estarán marcados por el acercamiento a los Estados Unidos en una fase económica desarrollista de exportación y sustitución de importaciones, que se conoce como "milagro económico colombiano" de Lleras Restrepo (Amézquita-Zárate, 2010). En lo social, durante el gobierno de Lleras Restrepo se crea el Ejército Popular de Liberación (EPL) (febrero de 1967) como brazo armado del Partido Comunista de Colombia Marxista Leninista y, como respuesta gubernamental, se fortalece al Ejército; la acusación de fraude en la elección de Pastrana llevó al surgimiento de la guerrilla urbana Movimiento 19 de Abril (M-19).

La estructura universitaria era sustancialmente diferente a las anteriores. Desde la década de 1940, la oferta de educación superior colombiana había coincidido con la fundación de 24 universidades, entre públicas (14) y privadas (10) (Jiménez \& Figueroa, 2000). Entre 1950 y 1967, hubo un aumento en el número de estudiantes. "Hacia 1974 había en Colombia 142.000 estudiantes matriculados: cerca de 75.500 en universidades públicas y 66.500 en universidades privadas" (Acevedo-Tarazona, 2015: 104), lo que suponía un $0,62 \%$ de la población del país. La primera reestructuración universitaria se produjo en la década de 1930, pero fue la segunda reforma modernizadora, en las décadas de 1960 y 1970, "[...] sin duda la más importante" (AcevedoTarazona, 2015: 103). Rodolfo de Low Maus fue decano de la Universidad Industrial de Santander (UIS) de 1962 a 1968 e introdujo el modelo americano de educación para el trabajo, además de convenios con la Fundación Ford y la Organización de las Naciones Unidas para la Educación, la Ciencia y la Cultura (UNESCO). En 1962 se crea el Fondo Universitario Nacional (FUN), que supone un aumento de la autonomía universitaria (Acevedo-Tarazona, 2012). De acuerdo con el programa estadounidense Alianza para el Fomento de la Cooperación con Aliados, Rudolph Atcon aterrizó en Colombia en 1964 portando el Plan Básico que sustentaba la reforma del Rector José Félix Patiño Restrepo en la Universidad Nacional de Colombia (UNAL). Los contenidos de Ciencias Sociales trajeron consigo la percepción de contradicciones y el refinamiento de la conciencia crítica y el fortaleciendo ideas de la élite intelectual de izquierda (Acevedo-Tarazona, 2015).

El ataque a la autonomía estudiantil se inició con la remoción del FUN, considerado ilegal, debilitando la estructura organizativa y asistencial a los estudiantes universitarios colombianos (Acevedo-Tarazona \& Gómez-Silva, 2000), perdiendo el control los estudiantes de la distribución de residencias, alimentos y asistencia médica, lo que llevó a las representaciones regionales a asumir el papel del movimiento estudiantil, como ocurrió en la UIS con Asociación Universitaria de Santander (AUDESA) (Acevedo-Tarazona \& Gómez-Silva, 2000). 
En 1969, los estudiantes protestaron por las injerencias de Estados Unidos en la política interna (Acevedo-Tarazona, 2015). Hubo disturbios en la UNAL y se cerró la Universidad de Antioquia (UdeA) en julio. En la Universidad de Caldas, hubo revuelta contra el hecho de que "[...] presidente Lleras al nombrar unilateralmente al rector" (Acevedo-Tarazona, 2015, p. 108) y varias manifestaciones contra los estadounidenses, con la expulsión de la Fundación Ford del Departamento de Sociología de la UNAL, así como un alboroto que involucró a estudiantes de la Universidad del Valle (UNIVALLE) que acusaron de espionaje a miembros del Cuerpo de Paz (Acevedo-Tarazona, 2015; CruzRodríguez; 2016; Sosa-Londoño, 2018).

En 1970, Misael Pastrana Borrero inauguró su gobierno bajo una tensión generalizada y denuncia de fraude electoral. Las protestas incluían a pueblos originarios, trabajadores y campesinos, además de estudiantes y profesores, con manifestaciones duramente reprimidas por las fuerzas policiales (Hernández-Arteaga, 2007). En febrero de 1971, la huelga de la UNIVALLE exigió la renuncia del decano, el retiro de la Iglesia y el sector privado del Consejo Universitario y el fin de la injerencia internacional en el otorgamiento de créditos educativos en la institución (Acevedo-Tarazona, 2015). En los enfrentamientos del 26 de febrero, "[...] un saldo de más de 20 muertos. De inmediato el gobierno declaró, por medio del decreto 250, el estado de sitio en todo el país" (Pardo \& Urrego, 2003, p. 1), sin embargo, lejos de pacificar, la crisis se agudizó.

Mediante el Decreto Legislativo 580, Pastrana Borrero garantizó la facultad de suspender actividades en instituciones educativas a nivel nacional (Acevedo-Tarazona, 2015; Acevedo-Tarazona \& Villabona-Ardila, 2015). El ministro de Defensa Nacional, Hernando Correa Cubides, llamó a los estudiantes "agitadores" y señaló los disturbios estudiantiles como parte de un plan para poner en peligro la realización de los Juegos Panamericanos, sugiriendo incluso el peligro de secuestro de deportistas extranjeros (Pardo \& Urrego, 2003).

Las autoridades convencieron a la opinión pública en contra de los jóvenes, acusándolos de ser guerrilleros entrenados por fuerzas externas para perturbar la paz de la nación (Pardo \& Urrego, 2003). Los estudiantes emitieron una nota exigiendo la revocación de contratos con extranjeros y una mayor inversión de recursos públicos para financiar la educación superior.

En el comunicado de la UNAL del 12 de marzo, ya contaban los principios del Programa Mínimo de los Estudiantes Colombianos, en el que participaron en la redacción estudiantes de 28 universidades (Acevedo-Tarazona, 2015). El documento recogía puntos relacionados con: la representación universitaria, autonomía, garantía de inversiones públicas, control de las tasas de matrícula en instituciones privadas, independencia en la investigación académica, injerencia en la elección de rectores, revisión de contratos con extranjeros, retiro de la Fundación Ford de la UNIVALLE, derecho a la organización política y reapertura de la Facultad de Sociología de la Pontificia Universidad Javeriana (Pardo \& Urrego, 2003).

Como respuesta, el gobierno de Pastrana Borrero mediante el Decreto 1259 de 25 de junio de 1971 "Por el cual se dictan medidas relacionadas con la conservación del orden público" amplió el poder plenipotenciario a los rectores, 
que podían castigar con sanciones académicas, administrativas y disciplinarias, pudiendo remover personal universitario (Rudas, 2019), gravado irónicamente como un "decreto rector-policía" (Pardo \& Urrego, 2003). El 5 de julio de ese año, es nombrado por el Gobierno el sexto Rector de la UNAL en 6 años, al ingeniero Santiago Fonseca Martínez. El 16 de agosto se reabre la universidad, utilizando el Rector el Decreto 1259 para "expulsar a numerosos estudiantes y destituir a profesores de izquierda" (Cote-Rodríguez, 2009, p. 434), desatando la furia del movimiento estudiantil (Rudas, 2019, p. 128). Los estudiantes convocan un "plebiscito", que contó con 32.000 votos (17.000 en Bogotá y 15.000 de las demás seccionales) y sentenció la expulsión del Rector y parte de sus colaboradores del Campus universitario (Pardo \& Urrego, 2003, p. 12). La presión llevó a un grupo de estudiantes (20 de septiembre) al despacho de la Rectoría y obligó a salir al Rector (Borrero-Cabal, 2008, p. 243). La respuesta gubernamental fue al día siguiente, cuando el Ejército y la Policía Militar llegan a la Universidad (Rudas, 2019, p. 128). Los estudiantes vuelcan y queman un coche del Ejército cerca de la Universidad y el Brigadier a cargo de la operación, Camacho Leyva, es apedreado en la Facultad de Derecho (Rudas, 2019, p. 128) antes de que tomara el control del Campus (EL TIEMPO $^{9}, 22$ de septiembre de 1971). Acevedo-Tarazona (2015) evaluó la crisis como "incontrolable", con una huelga general, lo que llevó al gobierno a emitir el Decreto 2070 de 23 de octubre, con nuevas medidas relacionadas con "la conservación de orden público", que garantizaba la experiencia de cogobierno de universidades a la comunidad estudiantil de Bogotá y Medellín, es decir, la participación de docentes y estudiantes en los Consejos Superiores de la UNAL, en noviembre de 1971, y en la UdeA, en enero de 1972 (AcevedoTarazona, 2015).

El logro sin precedentes de los movimientos estudiantiles de Colombia fue efímero, ya que en "[...] finales de mayo de 1972 regresaron los rectores del gobierno, y con ellos, la suspensión de la participación ampliada de estudiantes y profesores en los Consejos Superiores" (Acevedo-Tarazona, 2015, p. 109). El recorte de los fondos gubernamentales a los organismos universitarios y la reducción de la representación estudiantil en los Consejos de la UNAL y la UdeA debilitaron el movimiento hasta su final (Acevedo-Tarazona, 2015).

\section{Conclusiones}

Los contextos en los tres casos fueron diferentes (dictadura militar franquista, priísmo mexicano y turismo colombiano) y las reacciones y consecuencias también. El compromiso en 1968 de la lucha política por la democracia, las libertades civiles y la autonomía universitaria, característico de la historiografía de los movimientos estudiantiles en las sociedades española y mexicana, también lo vivieron los estudiantes colombianos que enfrentaron la violencia institucional de los gobiernos mientras luchaban por la democracia.

La aproximación en los datos de trabajos sistematizados que abordan los movimientos estudiantiles en España, México y Colombia, entre 1968 y 1971, remite a los hechos del emblemático de los movimientos estudiantiles del 1968, aunque algunos de ellos (en España) son previos al Mayo del 68 en Francia, y coetáneos de la Primavera de Praga. Así, si bien el movimiento contracultural iniciado por los estudiantes franceses repercutió entre los jóvenes y 
trabajadores en todos los continentes, clamando por libertades y democracia, las causas ya estaban presentes con anterioridad, aunque es cierto que a partir de ese momento los movimientos tendrán un referente. De esta forma, se observan puntos comunes relacionados con la cultura política humanista en los tres movimientos analizados.

El crecimiento del estudiantado en las universidades de los tres países, supuso también el fin de la elitización de la universidad, una extensión de la base social a las clases medias, una juventud de espíritu rebelde, ímpetu revolucionario e ideales democráticos, al tiempo que estas ideas prendía entre una parte del profesorado (reprimido en los tres casos). Es en el caso mexicano en el que se ve un apoyo decidido del PCM, mientras en España aparece una fragmentación política, y en Colombia también se observa una participación izquierdista.

La represión militar y policial es el instrumento utilizado en los tres casos por las instancias gubernativas (a pesar de las diferencias políticas), con cargas policiales, detenciones de límites y brutal represión ante las protestas estudiantiles, con culmen en la Matanza de Tlatelolco.

Los medios de comunicación de distinto signo ( $A B C$ de Madrid, El Tiempo de Bogotá o El Heraldo de México) se posicionan del lado de los gobiernos. Desde las agencias de inteligencia mexicana y colombiana el argumento va a ser la amenaza comunista y el uso de la represión para proteger a la sociedad y a los extranjeros en el país durante los eventos deportivos internacionales, al tiempo que se incrimina a extranjeros en los sucesos.

Se concluye proponiendo la continuación de las investigaciones sobre el objeto, incluida la actualización teórico-metodológica y la investigación sobre los nuevos movimientos estudiantiles de la década del 2000, así como otras categorías de análisis, como las tecnologías de la información y la comunicación y las redes sociales. El análisis de los procesos históricos en España, México y Colombia hacen avanzar el conocimiento apoyado en la transculturalidad y pueden servir a la investigación en Ciencias Sociales y Humanidades, especialmente en Historia, Geografía, Sociología, Antropología, Filosofía y Educación, entre otras.

\section{Referencias}

Acevedo-Tarazona, A. (2012). Modernización universitaria y protesta estudiantil en Colombia: el caso de la Universidad Industrial de Santander (19531977). Anuario de Historia Regional y de las Fronteras, 17(2), 571-599.

Acevedo-Tarazona, A. (2015). Educación, reformas y movimientos universitarios en Colombia: apuestas y frustraciones por un proyecto modernizador en el siglo XX. Revista de estudios sociales, (53), 102-111.

Acevedo-Tarazona, A., \& Gómez Silva, F.J. (2000). Conflicto y violencia en la universidad en Colombia. El proyecto modernizador y el movimiento estudiantil universitario en Santander, 1953-1980. Reflexión política, 2(4), $1-11$. 
Acevedo-Tarazona, A., \& Samacá-Alonso, G. (2011). Revolución y cultura en América Latina: el movimiento estudiantil como objeto de estudio en la historiografía colombiana y continental. Memoria y sociedad, 15(31), 104119.

Acevedo-Tarazona, A., \& Villabona-Ardila, J.V. (2015). (2015). El cogobierno en la Universidad de Antioquía, 1970-1973: una victoria del movimiento estudiantil y profesoral. Historia y Espacio, 11(44), 145-169.

Allier-Montana, M.E. (2009). Presentes-pasados del 68 mexicano: Una historización de las memorias públicas del movimiento estudiantil, 19682007. Revista mexicana de sociología, 71(2), 287-317.

Alyrio, R. D. (2009). Métodos e técnicas de pesquisa em administração. Rio de Janeiro: Fundação CECIERJ, 58-60.

Amézquita-Zárate, P. (2010). Aspectos económicos del gobierno de Carlos Lleras Restrepo (Colombia, 1966-1970). Revista Civilizar Empresa y Economía, 1(1), 36-53.

Argullol-Murgadas, R. (1977). Reflexión sobre los años radicales (movimiento estudiantil 1968-1971). Materiales, (2), 83-92.

Ayala-Diago, C. A. (2008). (2008). Exclusión, discriminación y abuso de poder en El Tiempo del Frente Nacional. Una aproximación desde el análisis crítico del discurso ( $A C D$ ). Universidad Nacional de Colombia.

Badenes-Salazar, P. (2018). Fronteras de papel: el mayo francés en la España del 68. Ediciones Cátedra.

Borrero-Cabal, A. (2008). La universidad: estudio sobre sus orígenes, dinámicas y tendencias. Editorial Pontificia Universidad Javeriana.

Carrillo-Linares, A. (2006). Movimiento estudiantil antifranquista, cultura política y transición política a la democracia. Pasado y Memoria, Alicante, 5. 149172.

Carrillo-Linares, A. (2008). Subversivos y malditos en la Universidad de Sevilla (1965-1977). Centro de Estudios Andaluces.

Carrillo-Linares, A. (2015). Universidades y transiciones políticas: el caso español en los años 60-70. Espacio, Tiempo y Educación, 2(2), 49-75.

Carrillo-Linares, A. (2018). El Mayo francés y España: impactos culturales y consecuencias políticas. Historia del presente, (31), 59-73.

Carrillo-Linares, A., \& Rodríguez-Tejada, S. (2019). 1968 y los estudiantes españoles: del antifranquismo a la revolución. In Las convulsiones del 68: España y el sur de Europa (pp. 29-63). UNED, Universidad Nacional de Educación a Distancia.

Carrillo-Linares, A., \& Cardina, M. (2012). Contra el Estado Novo y el Nuevo Estado. El movimiento estudiantil ibérico antifascista. Hispania, 72(242), 639-668.

Castillo-Troncoso, A. D. (2008). El movimiento estudiantil de 68 narrado en imágenes. Sociológica, 23(68), 63-114. 
Cejudo-Ramos, D. de J. (2020). La disputa por la Federación de Estudiantes de la Universidad de Sonora 1967-1968. Historia y problemas del siglo XX/ Año 11, Volumen 12, p. 36-52.

Cote-Rodríguez, J. (2009). (2009). El movimiento estudiantil de 1971: entre la homogeneidad y la heterogeneidad". Archila Neira, Mauricio, et. al. Una historia inconclusa: Izquierdas políticas y sociales en Colombia. Bogotá: Cinep-Colciencias-Programa por la Paz.

Cruz-Rodríguez, E. (2016). La izquierda se toma la universidad. La protesta universitaria en Colombia durante los años sesenta. Izquierdas, (29), 205232.

Dávila, L. R. (2021). Mayo 68 francés y el movimiento estudiantil. La imaginación al poder y los aparatos ideológicos del Estado. Revista Foro Cubano de Divulgación. 4(33), 1-13.

Del Castillo-Troncoso, A. (2008). El movimiento estudiantil de 1968 narrado en imágenes. Sociológica (México), 23(68), 63-114.

Delgado, K. S. (2013). El Partido Comunista Mexicano y el movimiento estudiantil de 1968: enfrentamiento, aportación e impacto. Unpublished thesis, Universidad Autónoma de Querétaro, Querétaro.

Díaz-Jaramillo, J. A. (2021). El movimiento estudiantil de 1971. La historia prohibida..., Revista CEPA, 1(31), 20-25.

Donoso-Romo, A. (2017). El movimiento estudiantil mexicano de 1968 en clave latinoamericana: aproximación a las nociones de educación y transformación social. Historia Crítica, (63), 137-157.

Feixa, C., \& Porzio, L. (2004). Los estudios sobre culturas juveniles en España (1960-2003). Revista de Estudios de Juventud, 2004, núm. 64, 9-28.

Flores-Padilla, G. (2021). Memoria e identidad. El movimiento estudiantil de 1968 y el archivo histórico de la Universidad Autónoma de México. Instituto de Investigaciones sobre la Universidad y la Educación. Archivo Histórico de la Universidad Nacional Autónoma de México. 1-21.

Fuentes, C. (2008). Em 68: Paris, Praga e México. Rio de Janeiro: Editora Rocco, 2008.

Gollás, M. (2003). México, crecimiento con desigualdad y pobreza:(de la sustitución de importaciones a los tratados de libre comercio con quien se deje) (No. 3). Centro de Estudios Económicos, El Colegio de México.

Hansen, R. D. (1998). La política del desarrollo mexicano. Siglo XXI.

Hernández-Arteaga, I. (2007). El programa mínimo de los estudiantes colombianos. Movimiento estudiantil universitario de 1971 por la universidad. Todo un país. Revista Historia de la educación colombiana, 10(10), 29-57.

Hernández-Ramos, P. (2017). Consideración teórica sobre la prensa como fuente historiográfica. Historia y comunicación social, 22(2), 465.

Jiménez, A., \& Figueroa, H. (2000). Políticas educativas en la educación superior: 1925-1992. Revista Colombiana de Educación, 38, 181-200. 
Jiménez-Guzmán, H. (2011). El 68 y sus rutas de interpretación: una crítica historiográfica (Doctoral dissertation, tesis de maestría, Universidad Autónoma de México).

Marini, R.M. (1970). Los movimientos estudiantiles en América Latina, un marco de referencias. Ciencia Social, Concepción, 110-122.

Marsiske, R. (2006). La Universidad de México: historia y desarrollo. Revista Historia de la Educación Latinoamericana, 8, 11-34.

Massholder, A.G. (2018). La recepción de la Revolución Cubana en los Partidos Comunistas de América Latina. Izquierdas, (42), 122-136.

Meyer, L. (2013). Nuestra tragedia persistente: la democracia autoritaria en México. Debate.

Pardo, M., \& Urrego, M. (2003). El movimiento estudiantil de 1971 en Colombia. In Primer Congreso Internacional sobre Historia de las Universidades de América y Europa.

Rahona-López, M.M. (2008). (2008). La educación universitaria en España y la inserción laboral de los graduados en la década de los noventa. Un enfoque comparado. Madrid, Premios Injuve para Tesis Doctoral.

Rodríguez-Tejada, S. (2011). Rodríguez Tejada, S. (2006). Dictadura franquista y movimiento estudiantil en la Universidad de Valencia (1939-1975). Universidad de Valencia.

Rudas, N. (2019). La violencia y sus resistencias en la universidad nacional de Colombia. Seis décadas de revolución y democracia en el campus. Sociología.

Ruiz, D. (Dir.) (1996). Historia de Comisiones Obreras (1958-1988). Siglo XXI.

Serna, A. M. (2014). La vida periodística mexicana y el movimiento estudiantil de 1968. Signos históricos, 16(31), 117-159.

Sierra-Garzón, F. A. (2015). La Política Educativa colombiana en el Gobierno de Carlos Lleras Restrepo (1966-1970). Reflexión Política, 17(33), 122-131.

Sosa-Londoño, Y.H. (2018). La protesta estudiantil en la Universidad del Valle entre 1968-1971 y 2007-2011. Una lectura comparada. In Forum. Revista Departamento de Ciencia Política, (13) 33-74

Suárez-Inclán, J., \& Gómez-Acebo (2021). Movimiento estudiantil en México y Uruguai: impulsos y deudas tras el 68. Instituto Universitario de Investigación en Estudios Latinoamericanos - Universidad de Alcalá. Documentos de Trabajo IELAT. N. 145, Abril 2021, 1-42.

Sucupira, T.G. (2021). (2021). Movimentos estudantis na UFC e ecos de resistência de 1968 e 2016. Tese Doutorado em Educação, Universidade Federal do Ceará.

Sucupira, T.G., \& García-Delgado, F.J. (2020). Reflexões metodológicas sobre a historiografia do movimento estudantil. Revista Educação \& Formação, 5(2), 216-239. 
Sucupira, T.G., García-Delgado, F.J., Vasconcelos, J.G., \& Matos, C.S.D. (2020). Um olhar nos movimentos estudantis do Brasil, México e Colômbia, entre 1968 e 1971. Revista Educação em Debate, 42, 81, 179-209.

Sucupira, T.G.; Vasconcelos, J.G.; Matos, C.S.D. (2019). O maio de 1968: Espanha e Fortaleza, Ceará. In: Seminário de Práticas Educativas, Memórias e Oralidades, 6., 2019, Fortaleza. Anais [...]. UECE, 2019, 915920.

Taborga-Torrico, H. (2003). Expansión y diversificación de la matrícula de la educación superior en México. Anuies.

Ysàs, P. (2007). ¿Una sociedad pasiva? Actitudes, activismo y conflictividad social en el franquismo tardío. Ayer, 31-57.

\section{Notas:}

\footnotetext{
' La base de este trabajo fue presentada en portugués a VII e VIII Seminário de Práticas Educativas Memórias e Oralidades (SEPEMO), da Universidade do Estado do Ceará (UECE), de 26 a 28 de agosto de 2021 (online).

${ }^{2}$ Este artículo es el resultado de la investigación realizada durante la pasantía de doctorado de Tânia Gorayeb Sucupira en la Universidad de Huelva (UHU), España, entre agosto de 2019 y enero de 2020, financiada por la Coordenação de Aperfeiçoamento de Pessoal de Nível Superior (CAPES), bajo la supervisión del Dr. Francisco Javier García-Delgado, Profesor Titular de Análisis Geográfico Regional.

${ }^{3}$ El uso de esta lengua se debe al objetivo de desarrollo de la competencia lingüística dentro de la pasantía de doctorado realizada en la UHU.

${ }^{4}$ Comisiones Obreras (CC.OO.) es un movimiento sindical de orientación comunista, que tiene su origen en 1958, en contra del régimen franquista, y que fue duramente reprimido durante la dictadura (Ruiz, 1993). Actualmente es uno de los grandes sindicatos del país (junto con la Unión General de Trabajadores, de orientación socialista), contando con alrededor de 1 millón de afiliados y miles de representantes sindicales en la administración pública y empresas privadas.

${ }^{5}$ Catedrático de Historia de América, detentó el mandato de Rector de 1969 a 1971.

6 Creado en 1903, es un periódico diario de orientación conservadora, monárquica y católica. Tradicionalmente tuvo una edición en Madrid y otra en Sevilla.

7 Este partido, con diferentes denominaciones, gobernó ininterrumpidamente el país desde 1930 (con Pascual Ortiz Rubio) hasta 2000 (Ernesto Zedillo Ponce de León), pasando por diferentes espectros ideológicos.

${ }^{8}$ Fundado en 1965 (Serna, 2014), estaba dirigido por el empresario poblano Gabriel Alarcón, muy cercano a Díaz Ordaz (del Castillo-Troncoso, 2008).

${ }^{9}$ Principal diario colombiano, creado en 1910 , de ideología centrista, vinculado a la familia Restrepo, apoyó el Frente Nacional y el turnismo político (Ayala-Diago, 2008).
} 